\title{
PERCEPÇÃO DA EQUIPE DE ENFERMAGEM EM RELAÇÃO A UM NOVO MODELO DE QUADRO BEIRA LEITO DE IDENTIFICAÇÃO E COMUNICAÇÃO SOBRE O PACIENTE EM UNIDADE DE TERAPIA INTENSIVA \\ PERCEPTION OF THE NURSING TEAM IN RELATION TO A NEW BED BEDROOM FRAMEWORK MODEL FOR IDENTIFICATION AND COMMUNICATION ABOUT THE PATIENT IN THE INTENSIVE CARE
}

SILVA, Eloise Natane ${ }^{1}$; RIBEIRO, Cristina A. P. Silva ${ }^{2}$; VERISSIMO, Vanda A. C. Rivera ${ }^{3}$; ${ }^{1}$ Pós Graduanda do Programa de Residência Multiprofissional em Enfermagem Intensiva Universidade São Francisco; ${ }^{2}$ Tutora de Residência Multiprofissional em Saúde Intensiva em Enfermagem - Universidade São Francisco; ${ }^{3}$ Preceptora de Residência Multiprofissional em Saúde Intensiva em Enfermagem - Universidade São Francisco

\section{natane.eloise@icloud.com}

RESUMO. A internação em Unidade de Terapia Intensiva, implica em um cuidado capacitado e sistemático, uma vez que sua rotina exige alta tecnicidade, sendo considerado um cenário de alto risco, além disso internações neste ambiente são consideradas críticas, com eminente risco de morte, que requerem ações rápidas e precisas, além de inúmeros procedimentos. Justamente por sua peculiaridade assistencial os riscos de eventos adversos encontram-se exacerbado. Assim faz-se necessário a elaboração de estratégias alternativas para garantir a segurança do paciente, qualidade assistencial e um cuidado livre de danos. Frente às características singulares presentes na prática clínica das Unidades de Terapia Intensiva, que muitas vezes, são fatores que implicam na segurança do paciente, como na prática de identificação e comunicação, este estudo teve como objetivo avaliar a percepção da equipe de enfermagem frente a um novo modelo de quadro beira leito de identificação e comunicação sobre o paciente, implementado em uma Unidade de Terapia Intensiva de um Hospital Universitário do interior de São Paulo. O trabalho tratou-se de uma pesquisa de campo, exploratória, de natureza quantitativa. Os resultados encontrados evidenciaram que na percepção da amostra o quadro se faz efetivo ao que se propõe em identificar os pacientes e realizar a comunicação sobre o mesmo, principalmente no que se diz respeito aos riscos que este está exposto, as boas práticas, e dispositivos em uso. Porém a segurança do paciente, relacionada à sua correta identificação e comunicação somente será bem-sucedida com o comprometimento das equipes envolvidas nas ações descritas nas rotinas dos protocolos.

Palavras-chave: enfermagem, segurança do paciente, identificação do paciente.

ABSTRACT. Hospitalization in the Intensive Care Unit implies skilled and systematic care, since its routine requires high technicality, being considered a high-risk scenario. Furthermore, hospitalizations in this environment are considered critical, with an imminent risk of death, which require fast and accurate actions, in addition to numerous procedures. Precisely because of its care peculiarity, the risks of adverse events are exacerbated. Thus, it is necessary to develop alternative strategies to ensure patient safety, quality of care and damage-free care. In view of the unique characteristics present in the clinical practice of Intensive Care Units, which are often factors that affect patient safety, such as the practice of identification and communication, this study aimed to assess the perception of the nursing team towards a new bedside model for patient identification and communication, 
implemented in an Intensive Care Unit of a University Hospital in the interior of São Paulo. The work was an exploratory field research of a quantitative nature. The results found evidenced that, in the sample's perception, the framework is effective in what it proposes to identify patients and carry out communication about it, especially with regard to the risks it is exposed to, good practices, and devices in use. However, patient safety, related to their correct identification and communication, will only be successful with the commitment of the teams involved in the actions described in the protocol routines.

Keywords: nursing. patient safety, patient identification.

\section{INTRODUÇÃO}

Desde os primórdios, sabe-se que qualquer tipo de cuidado prestado, pode vir a causar algum dano ao paciente. Ao longo da história, diversos personagens contribuíram com a melhoria da qualidade assistencial em saúde, criando métodos que diminuem a exposição do paciente ao risco e assim menor incidência de eventos adversos.

As atividades em prol a segurança do paciente, e estratégias para a diminuição de eventos adversos, deram-se início nos anos 90, após a publicação do relatório do Institute of Medicine dos Estados Unidos da América, que apresentou resultados críticos sobre a situação da assistência à saúde daquele país (SILVA, 2010).

Um movimento considerado essencial, e que impactou diretamente no Brasil, ocorreu em 2004, com a criação da Aliança Mundial para Segurança do Paciente. O Brasil é um dos países signatários do acordo, cujo objetivo é aplicar os protocolos de segurança para melhorar a efetividade, eficiência e equidade. Com a Aliança, a Organização Mundial da Saúde (OMS) propõe 6 (seis) metas globais para alcançar a qualidade do atendimento. São elas: identificação correta dos pacientes; melhorar a comunicação entre os profissionais de saúde; melhorar a segurança na prescrição, no uso e na administração de medicamentos; assegurar cirurgia em local de intervenção, procedimento e paciente correto; higienizar as mãos para evitar infecções; e redução do risco de quedas e lesões de pele (WHO, 2009).

Ao tratarmos de qualidade assistencial, inúmeras vezes iremos nos deparar com a temática segurança do paciente, visando garantir e assegurar que o cuidado prestado proceda de forma eficiente, livre de erros e falhas e que estejam dentro dos padrões de qualidade estabelecidos (BRASIL, 2011).

Além disso, para que o paciente receba uma assistência de excelência, é imperativa a aplicação do gerenciamento de risco. Gerenciamento de risco é a aplicação sistemática de políticas de gestão, procedimentos, condutas e ações, para análise, avaliação, controle e monitoramento de risco e eventos adversos, de forma sistemática e contínua, que afetam a segurança, a saúde do paciente e, consequentemente, a imagem institucional (BRASIL, 2013).

A equipe de enfermagem tem grande influência na promoção da segurança do paciente pelo maior contingente de profissionais da área de saúde no Brasil e no mundo e por atuar em cenários de elevada complexidade e exposição a riscos, composto de contínuas interações entre pessoas, instalações, equipamentos e medicamentos. No entanto, o modelo de atenção à saúde exige um processo de trabalho cooperativo em que o enfermeiro deve ser ativo, líder e voltado para os cuidados de saúde, assumindo atitudes, dentre as quais está a motivação pela busca do conhecimento com vistas a melhorar o cuidado seguro ao paciente e à população (BEZERRA, 2018). 
Em Unidades de Terapia Intensiva, encontramos características totalmente diferentes de outras unidades de internação. É um ambiente cuja dinâmica impõe ações profundas, necessitando de atenção e mão de obra especializada, em sua maioria são ambientes destinados ao atendimento de pacientes graves, com potencial risco de morte, que necessitam de atendimento ininterrupto, acredita-se ainda que quando o cuidado se dá em um ambiente que exige alta tecnicidade, tal como ocorre em uma unidade de terapia intensiva (UTI), o cuidar pode tornar-se mecânico devido à alta complexidade de equipamentos, tecnologia e nível de consciência dos pacientes, o que pode favorecer para o aumento de riscos. Assim, a internação em unidades de alta complexidade implica em uma situação de grande estresse para todos os envolvidos. Frente a isso, faz-se necessário uma gestão de riscos rigorosa e sistemática, uma vez que estes pacientes estão expostos a inúmeros fatores.

A internação na UTI rompe bruscamente com o modo de viver do paciente, pois nessas unidades destacam-se aqueles que apresentam o rebaixamento do nível de consciência, caracterizado pela ausência de respostas verbais e não-verbais. Apesar da sedação ser uma excelente aliada nos tratamentos necessários para o paciente de terapia intensiva, devemos considerar que ao alterarmos o nível de consciência do mesmo, ocorre também uma alteração no relacionamento interpessoal entre este paciente e equipe de enfermagem, consequentemente afeta a comunicação deste indivíduo e assim o mesmo não é capaz de realizar a confirmação verbal de seus dados, como nome por exemplo, gerando um aumento no risco de eventos adversos relacionados a identificação do paciente (NASCIMENTO et al., 2004).

Ao tratarmos de ações para a diminuição de agravos e riscos relacionados a identificação do paciente, a identificação beira leito é uma ferramenta muito utilizada na prática intra hospitalar, em especial em unidades de terapia intensiva, e possui como principal objetivo fornecer de forma prática a confirmação do paciente ali internado. Uma outra forma, muito comum no ambiente intra-hospitalar é a pulseira de identificação. Independente do instrumento escolhido e utilizado pela instituição, este deve ser capaz de identificar corretamente o indivíduo como sendo a pessoa para a qual se destina o serviço (medicamentos, sangue ou hemoderivados, exames, cirurgias e tratamentos), como estabelece uma das principais metas da segurança do paciente: "Identificação correta dos pacientes". Em geral, esse processo exige pelo menos dois diferentes parâmetros, tais como como nome completo e data de nascimento ou número de identificação do prontuário, ou nome completo da mãe.

Ainda, a comunicação efetiva e o trabalho da equipe multiprofissional são compreendidos como determinantes da qualidade e da segurança na prestação de cuidados aos indivíduos As falhas na comunicação entre os profissionais de saúde têm sido um dos principais fatores que contribuem para a ocorrência de eventos adversos e, consequentemente, diminuição da qualidade dos cuidados. Os profissionais prestadores de cuidados de saúde têm dificuldades de manter uma comunicação que favoreça o trabalho em equipe e a continuidade dos cuidados em saúde intra e extra-hospitalar (OLINO et al., 2019).

Frente às características singulares presentes na prática clínica das Unidades de Terapia Intensiva, que muitas vezes, são fatores que implicam na identificação do paciente, e na comunicação da equipe, ocorrendo assim um aumento considerado de exposição aos riscos, houve a necessidade de criar um novo instrumento de identificação e comunicação sobre o paciente, além disso, mais do que implementar um novo instrumento, realizamos uma análise quanto sua efetividade em contribuir para a qualidade assistencial, através comunicação ao realizar a exposição de riscos e boas práticas. Este estudo teve, portanto, o 
objetivo de analisar a percepção da equipe de enfermagem frente a um novo instrumento de identificação e comunicação proposto, como também a qualidade do mesmo, ao que se refere em contribuir para a qualidade assistencial.

\section{METODOLOGIA}

Tratou-se de uma pesquisa de campo de intervenção, exploratória, de natureza quantitativa. A população de estudo foi composta por 42 profissionais da equipe de enfermagem, sendo 32 técnicos de enfermagem e 10 enfermeiros, atuantes em uma Unidade de Terapia Intensiva Adulto de um determinado Hospital Universitário no interior de São Paulo. Os critérios não inclusão da amostra foram os funcionários que estavam de férias, folga, ou afastados por licença médica, no período da coleta de dados.

Anteriormente a coleta de dados foi realizado uma intervenção, com auxílio de um "quadro de identificação e comunicação sobre o paciente" pré elaborado, fixado em beira leito de 36 pacientes, contendo: nome; data de nascimento; idade; data de admissão; dieta; enfermeiro (responsável pelo plantão); técnico de enfermagem (responsável pelo cuidado no plantão); classificação de risco, e boas práticas (frente ao risco evidenciado); atenção; recomendações / pendências; dispositivos, data de inserção, e data de troca; metas e condutas para o dia. Os quadros ficaram expostos por um período de 4 a 6 dias, sendo atualizado diariamente pela pesquisadora e enfermeiros de cada plantão.

Para a coleta de dados foi utilizado um questionário pré-elaborado, com 12 questões de múltipla escolha, com assuntos de cunho social e profissional; referentes ao conhecimento da equipe em relação a segurança do paciente; e a percepção dos participantes sobre o quadro de identificação e comunicação sobre o paciente. Este questionário foi elaborado utilizando informações contidas em bases de dados como biblioteca eletrônica Scientific Electronic Library Online (SCIELO), livros e revistas através dos seguintes descritores: identificação do paciente, segurança do paciente, enfermagem, classificação de risco.

Os dados obtidos através do questionário aplicados a equipe de enfermagem foram tabulados e analisados estatisticamente, e apresentados de forma gráfica, com números percentuais e absolutos. Os mesmos foram agrupados para discussão, comparando-os com a literatura. Este trabalho teve a aprovação do Comitê de Ética CAEE $n^{\circ}$ 50145021.9.0000.5514.

\section{RESULTADOS E DISCUSSÃO}

Durante este trabalho, após a intervenção com o quadro de identificação e comunicação (Figura 1), foram entrevistados 42 profissionais da equipe de enfermagem, sendo técnicos de enfermagem e enfermeiros, todos atuantes em uma Unidade de Terapia Intensiva Adulto, de um Hospital Universitário localizado no interior paulista.

Conforme representado na Tabela 1 , destes 42 profissionais, 32 são técnicos de enfermagem o que representa um total de $76 \%$ da amostra, e $24 \%$ são enfermeiros.

Segundo pesquisa do Conselho Nacional de Enfermagem, o Cofen em conjunto com a Fiocruz, a categoria da enfermagem, onde há auxiliares, técnicos de enfermagem e enfermeiros, conta com 1,6 milhão de profissionais. Atualmente, a enfermagem no país é composta por um quadro de $80 \%$ de técnicos e auxiliares e $20 \%$ de enfermeiros. Mais da metade dos enfermeiros $(53,9 \%)$, técnicos e auxiliares de enfermagem $(56,1 \%)$ se concentram na região Sudeste (FIOCRUZ, 2015). 
Dentro das Unidades de Terapia Intensiva, o paciente necessita de cuidados ininterruptos, 24 horas por dia, e em sua maioria são aqueles que vão desde cuidados básicos do dia a dia como higiene corporal e alimentação, até cuidados que requerem mais tecnicidade como procedimentos médicos hospitalares específicos. Assim o tempo de permanência dos profissionais da equipe de enfermagem com o paciente é considerado maior em relação aos outros profissionais da área da saúde, além disso, a área de saúde se compõe de um contingente de 3,5 milhões de trabalhadores, dos quais cerca 50\% atuam na enfermagem, tornando-se a segunda maior categoria do brasil, ficando atras apenas da classe dos metalúrgicos (FIOCRUZ, 2015).

Enfermeiro intensivista é o profissional especializado em cuidados de pacientes internados na UTI. Suas competências englobam cuidados de pacientes com necessidade de terapias avançadas de suporte de vida como ventilação mecânica, hemodiálise, administração contínua de medicamentos, e procedimentos privativos do enfermeiro, como passagem de sondas, punções arteriais, entre outros, além da supervisão da equipe e atividades gerenciais da unidade. O Técnico de enfermagem é o membro da equipe de enfermagem responsável pelos cuidados básicos do paciente, como higiene, a alimentação, a administração de medicamentos e a monitorização de funções vitais (COFEN,1987)

Em relação ao tempo de trabalho desses profissionais na categoria (técnico de enfermagem ou enfermeiro) dentre os entrevistados, foi possível observar que: $33 \%$ atuam a mais de 10 anos na enfermagem, $19 \%$ de 05 a 10 anos, e $48 \%$ possuem menos de 5 anos de atuação na área. E ao tempo de trabalho no setor onde ocorreu o estudo $9,5 \%$ possuem mais de 10 anos de atuação, $19 \%$ de 5 a 10 anos, e $71,5 \%$ menos de 5 anos no setor (Tabela 1). Nota-se que grande maioria desses profissionais entrevistados estão a pouco tempo atuando neste setor, menos de 5 anos, podemos relacionar este fato ao grande número de contratações que ocorreu entre o segundo semestre de 2019 a 2021, devido a pandemia da COVID 19, onde houve um aumento na demanda de internações hospitalares e mais do que nunca necessidade dos serviços prestados pela equipe de enfermagem.

Conforme dados do Cadastro Geral de Empregados e Desempregados (CAGED) do Governo Federal, coletados entre janeiro e junho de 2020, o número de contratações de enfermeiros nos primeiros 6 meses desse ano foi 50,73\% maior do que no mesmo período de 2019. Comparando-se o saldo de contratações e demissões, essa diferença sobe para $155,3 \%$ (BRASIL, 2020).

Tabela 1 - Caracterização da amostra estudada segundo categoria profissional, tempo de trabalho na instituição de estudo e tempo de trabalho no setor de atuação $(\mathrm{N}=42)$.

\begin{tabular}{lcc}
\hline Categoria profissional & $\mathbf{N}$ & \% \\
\hline Enfermeiros & 10 & 24 \\
Técnicos de Enfermagem & 32 & 76 \\
Tempo de atuação na área & $\mathbf{N}$ & $\%$ \\
Menos de 01 ano & 3 & 7 \\
01 a 03 anos & 12 & 29
\end{tabular}

Cont. Tabela 1 


\begin{tabular}{lll}
\hline Cont. Tabela 1 & $\mathbf{N}$ & $\mathbf{\%}$ \\
\hline Tempo de atuação na área & 05 & 12 \\
03 a 05 anos & 08 & 19 \\
05 a 10 anos & 14 & 33 \\
Mais de 10 anos & $\mathbf{N}$ & $\mathbf{\%}$ \\
\hline Tempo de atuação no setor & 18 & 43 \\
\hline Menos de 01 ano & 08 & 19 \\
01 a 03 anos & 04 & 9,5 \\
03 a 05 anos & 08 & 19 \\
05 a 10 anos & 04 & 9,5 \\
Mais de 10 anos & & \\
\hline
\end{tabular}

Fonte: Próprio autor.

\section{IDENTIFICAÇÃO E COMUNICAÇÃO SOBRE O PACIENTE EM UTI ADULTO}

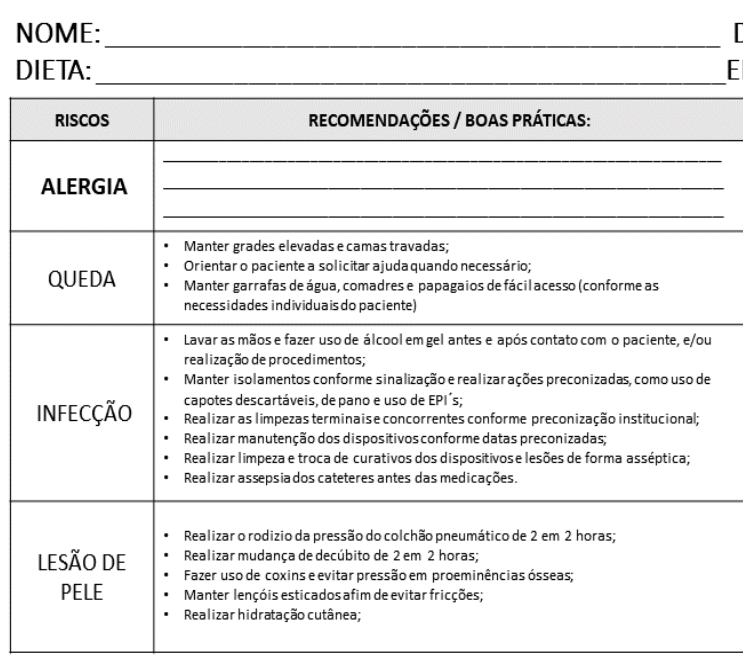

DN: ENFo

ENFo- ___ T

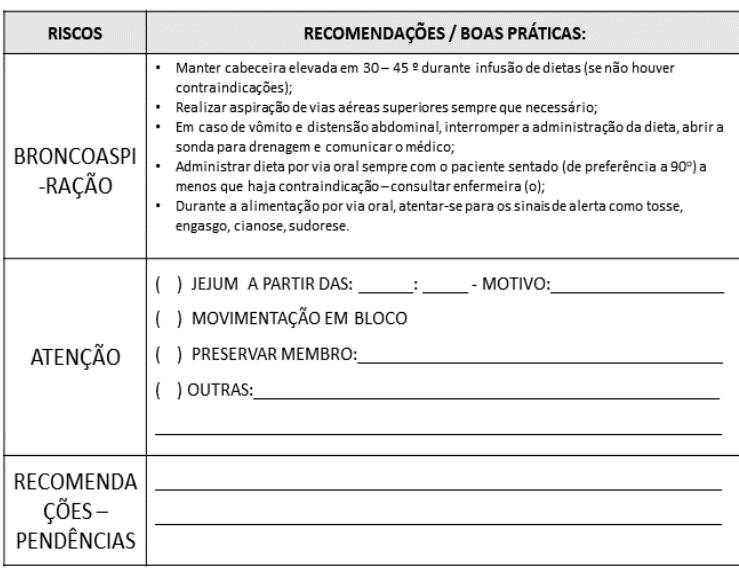

\begin{tabular}{|c|c|c|c|}
\hline DISPOSITIVOS & DATA DE INSERÇÃ̃o & DATA DE TROCA & METAS E CONDUTAS PARA HOJE \\
\hline 01 - & 01 - _ _ _ _ & 01. & \\
\hline $02-$ & 02. & $02-\ldots 1$ & \\
\hline 03 - & $03-\ldots$ & $03-\ldots$ & \\
\hline 04 - & 04. & $04-1 / 1$ & \\
\hline $05-$ & 05. & $05-\ldots$ & \\
\hline $06-$ & $06-\ldots$ & $06-\ldots$ & \\
\hline $07-$ & $07-\ldots$ & $07-\ldots$ & \\
\hline $08-$ & $08-\ldots$ & $08-\ldots$ & \\
\hline
\end{tabular}


Figura 1 - Imagem representativa do quadro utilizado beira leito durante a fase de intervenção da pesquisa "Quadro de Identificação e Comunicação sobre o Paciente em UTI Adulto" (Fonte: Próprio autor).

Na Tabela 2 é possível observar os dados coletados em relação ao conhecimento dos profissionais entrevistados sobre a temática segurança do paciente, onde através de uma pergunta de múltipla escolha foi questionado sobre o qual seria o conceito de segurança do paciente, e 95\% destes profissionais responderam de forma correta, e apenas $5 \%$, ou seja, 2 profissionais responderam de forma incorreta.

A OMS, por meio da Classificação Internacional de Segurança do Paciente define como conceito de segurança do paciente as ações promovidas pelas instituições de saúde para reduzir a um mínimo aceitável, o risco de dano desnecessário associado ao cuidado de saúde (WHO, 2009).

Segundo uma pesquisa inédita realizada pela Fiocruz (2015):

O desejo de se qualificar é um anseio do profissional de enfermagem. Os trabalhadores de nível médio (técnicos e auxiliares) apresentam escolaridade acima da exigida para o desempenho de suas atribuições, com 23,8\% reportando nível superior incompleto e $11,7 \%$ tendo concluído curso de graduação. O programa Proficiência e outras iniciativas de aprimoramento promovidas pelo Sistema Cofen/Conselhos Regionais revelaram ampla penetração, alcançando 94,5\% dos enfermeiros e $98 \%$ dos profissionais de nível médio (técnicos e auxiliares) que relatam participação em atividades de aprimoramento (Fiocruz, 2015).

Em relação as 6 (seis) metas propostas pela OMS (Organização Mundial de Saúde), para obter a qualidade na assistência em saúde, foi questionado aos profissionais quais seriam essas seis metas, por meio de uma pergunta de múltipla escolha, e dentre o total da amostra, 28 participantes, ou seja, $67 \%$ responderam de forma correta e 33\% de forma incorreta.

As 6 (seis) metas de segurança do paciente são: identificação correta dos pacientes; melhorar a comunicação entre os profissionais de saúde; melhorar a segurança na prescrição, no uso e na administração de medicamentos; assegurar cirurgia em local de intervenção, procedimento e paciente correto; higienizar as mãos para evitar infecções; e redução do risco de quedas e lesões de pele (BRASIL,2021).

A saúde incorpora novas tecnologias de uma forma muito rápida, todos os anos novos materiais e equipamentos são adquiridos. As tecnologias oriundas da segurança do paciente não devem ser direcionadas apenas aos gestores, mas dizem respeito também a todos da equipe. Os estabelecimentos de Saúde necessitam incorporar as novas tecnologias em um programa de educação permanente e continuada profissionais da Saúde, dessa forma eles continuaram atualizados. (BRASIL, 2014).

Tabela 2 - Conhecimento da amostra segundo a temática segurança do paciente e as 06 metas de segurança do paciente $(\mathrm{N}=42)$.

Conceito Segurança do Paciente

CORRETA

INCORRETA

Quais são as seis metas de segurança do paciente

N \%

40

95

2

5

$\mathbf{N}$
$\%$ 


\begin{tabular}{lll}
\hline CORRETA & 28 & 67 \\
INCORRETA & 14 & 33 \\
\hline
\end{tabular}

Fonte: Próprio autor.

Conforme evidenciado na Figura 2, os profissionais entrevistados, foram questionados se consideram que o quadro de identificação e comunicação sobre o paciente uma ação que contribui para a melhoria na assistência de enfermagem, e 98\% dos profissionais declararam considerar o quadro uma ação positiva para contribuir na qualidade da assistência, e apenas $2 \%$, um profissional declararam não ser uma ação que contribui.

Um quadro de identificação e comunicação sobre o paciente, tem como principal objetivo evidenciar de uma forma facilitada e clara informações importantes para a prática clínica, como nome, data de nascimento, riscos, boas práticas, entre outras informações pertinentes que visam à redução de eventos adversos relacionados à assistência. A identificação correta do paciente é muito importante para garantia da segurança do processo assistencial em qualquer situação, principalmente naquelas em que o paciente não pode responder por si mesmo, isso garante o atendimento correto para a pessoa correta. Além disso, a placa ou quadro de identificação faz parte de uma estratégia barata e prática, capaz de provocar mudanças na prática diária, e criar as barreiras necessárias para os eventos adversos em saúde.

A implementação efetiva de um protocolo de identificação de pacientes é um desafio institucional. Estratégias devem ser criadas para favorecer a adesão à rotina, e o uso de indicadores deve fazer parte do protocolo, conforme sugerido pelo Ministério da Saúde, para ajudar nas estratégias adotadas a cada reavaliação das rotinas implementadas (LEMOS, 2017) 


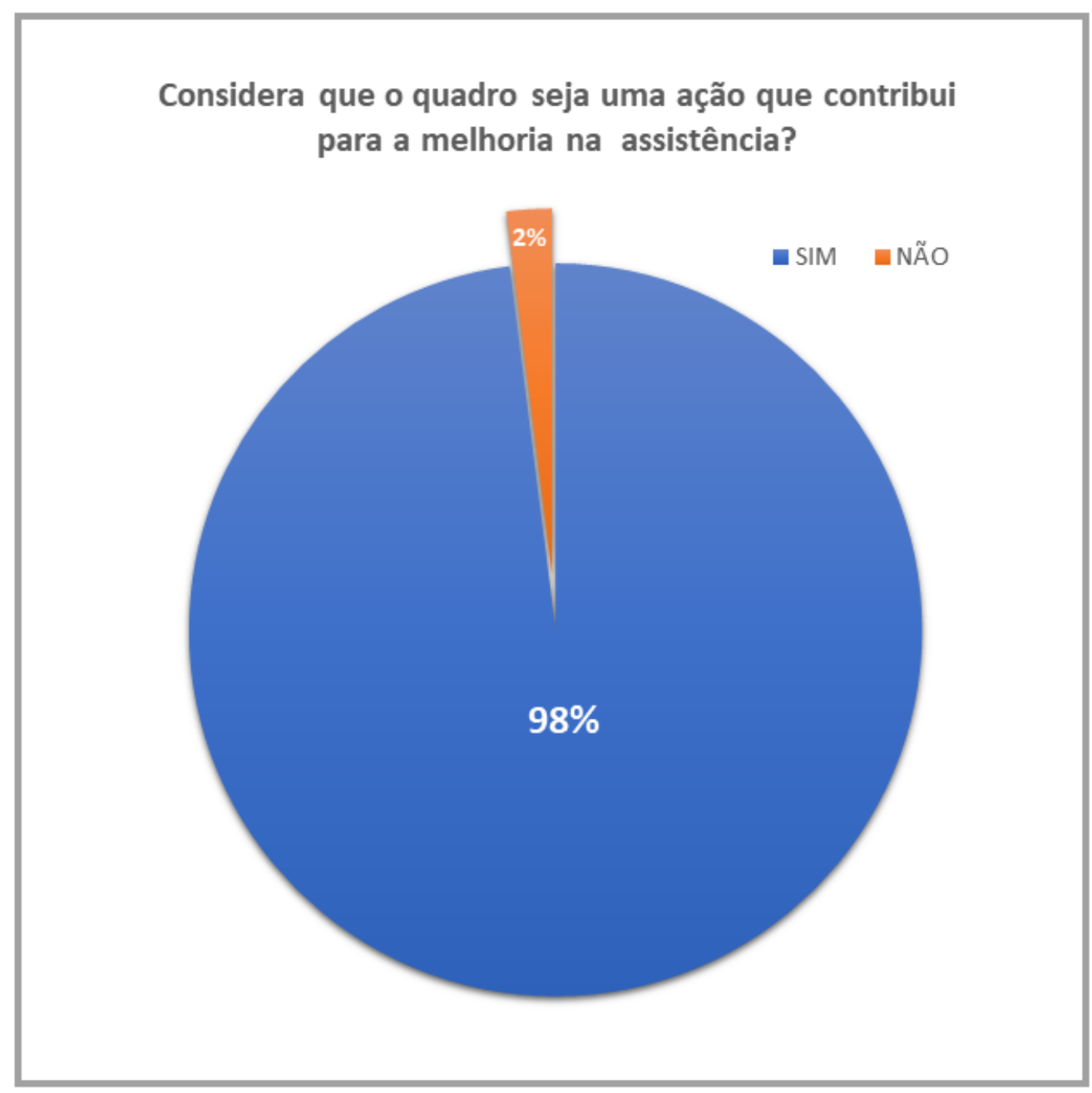

Figura 2 - Gráfico referente a opinião da equipe entrevistada em relação ao quadro de identificação e comunicação sobre o paciente (Fonte: Próprio Autor)

A última informação analisada foi a percepção da amostra quanto a eficácia do quadro estudado. Estes foram questionados em relação aos seus componentes de forma individual, e como podemos ver na Figura 3, 38 profissionais declaram que o quadro se faz muito eficaz ao que se refere a identificação do paciente, e 04 declaram ser eficaz; em relação aos riscos 30 profissionais declaram ser muito eficaz, 11 eficaz, e apenas 1 profissional declara ser pouco eficaz; ao evidenciar as boas práticas, 32 profissionais declaram ser muito eficaz, 7 eficaz, 2 pouco eficaz e 1 ineficaz; ao que se refere aos dispositivos, sua data de inserção e validade 32 declaram ser muito eficaz, 9 eficaz, e 1 pouco eficaz; referente as pendências 29 referem ser muito eficaz, 9 eficaz, 2 pouco eficaz e 2 ineficaz; e por último ao expor as metas e condutas para aquele dia, 25 deles declaram ser muito eficaz, 15 eficaz, 1 pouco eficaz e 1 ineficaz.

Ainda, durante a aplicação do quadro, foi levantado por alguns participantes a sugestão de inclusão de um campo destinado para lesões de pele, inserindo os tópicos em uso no tratamento dessas lesões, como também o período de troca dos curativos e condutas em relação a essas terapias, uma vez que o número de lesões por pressão em Unidades de Terapia Intensiva tem alta incidência. 
Diariamente, a comunicação na instituição de saúde é item básico e mais do que importante. Vale a pena frisar que a transmissão de uma mensagem nesse tipo de ambiente não é apenas de forma verbal, mas por meios oportunos e acessíveis a todos, sendo essa de forma precisa, completa, oportuna e compreendida por todos. Dessa forma, as instituições de saúde reduzem a ocorrência de erros, resultando na melhoria da segurança do paciente.

Melhorar a comunicação interna nas instituições de saúde é promover a segurança e bem-estar dos pacientes, por isso, é tão importante investir em meios alternativos, como por exemplo os quadros de comunicação beira leito, para que tornem esse processo cada vez mais prático e eficaz.

A comunicação eficiente faz parte das seis Metas Internacionais de Segurança do Paciente. A meta 2 "melhorar a comunicação entre os profissionais de saúde", orienta que o hospital deve "evitar que informações sobre os cuidados do paciente sejam perdidas ou trocadas, melhorando a efetividade da comunicação entre os profissionais da saúde"(BRASIL, 2013)

A comunicação efetiva e o trabalho da equipe multiprofissional são compreendidos como determinantes da qualidade e da segurança na prestação de cuidados aos indivíduos' As falhas na comunicação entre os profissionais de saúde têm sido um dos principais fatores que contribuem para a ocorrência de eventos adversos e, consequentemente, diminuição da qualidade dos cuidados. Os profissionais prestadores de cuidados de saúde têm dificuldades de manter uma comunicação que favoreça o trabalho em equipe e a continuidade dos cuidados em saúde intra e extra-hospitalar, seja por falta de tempo, escassez de pessoal, ausência de padronização, imperícia ou desconhecimento da importância de tal ação (OLINO et al., 2019) 


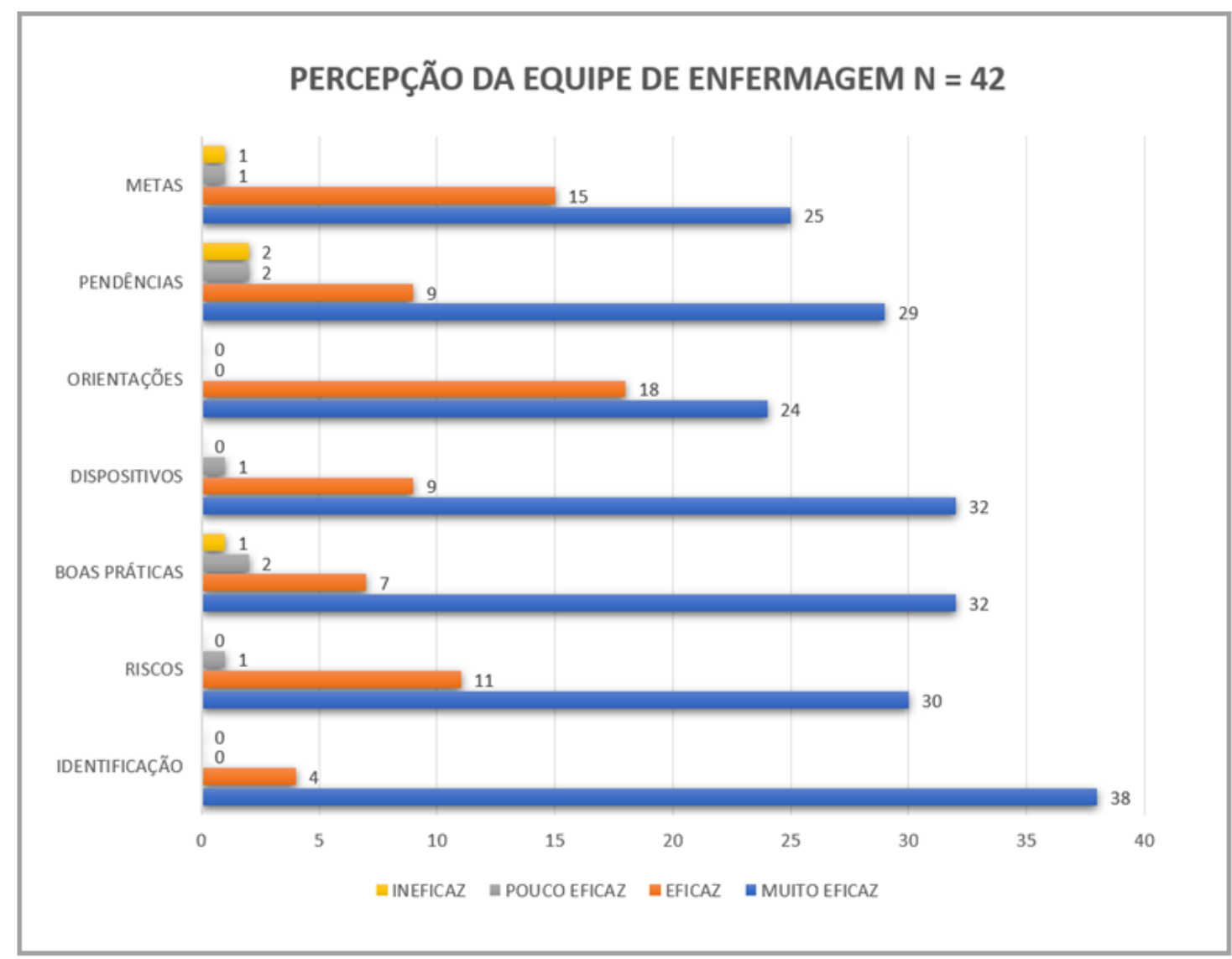

Figura 3 - Gráfico referente a percepção da equipe entrevistada em relação ao quadro de identificação e comunicação sobre o paciente de forma individual a cada componente (Fonte: Próprio Autor).

\section{CONCLUSÃO}

A presente pesquisa permitiu analisar uma estratégia para promover a segurança do paciente no contexto hospitalar, ao que se refere a identificação e comunicação sobre o paciente. Por meio dos dados coletados e discussão levantada acima, foi possível concluir que os participantes apresentaram conhecimentos limitados em relação a temática "Segurança do Paciente", demostrando assim a necessidade de uma educação permanente e continuada, afim de se reciclar informações, e ofertar de forma oportuna novos conhecimentos aos colaboradores. Ao que se refere ao quadro de identificação e comunicação proposto, este se mostrou efetivo ao que se refere na identificação do paciente; riscos e boas práticas; e dispositivos e suas validades.

O estudo teve como limitação a descontinuidade do preenchimento do quadro pelos enfermeiros, pois houve casos em que não ocorreu a atualização do quadro em tempo hábil e oportuno, assim se perdendo algumas informações, concluindo que há necessidade de atualização a cada troca de plantão, admissão ou alta dos pacientes. Para que isto ocorra é necessário que a habilidade de comunicação seja sempre desenvolvida e treinada periodicamente para que todos possam compreender e colocar em prática o método escolhido. A segurança do paciente, relacionada à sua correta identificação e comunicação somente será 
bem-sucedida com o comprometimento das equipes envolvidas nas ações descritas nas rotinas dos protocolos.

Não obstante, traz contribuições a prática clínica, ao passo que desperta o conhecimento sobre a temática e impacta no interesse dos entrevistados ao procurar mais sobre a temática segurança do paciente. Também contribui para a pesquisa, pois divulga uma experiência frente as estratégias alternativas para melhorar na identificação do paciente e na comunicação efetiva, sendo esta fundamental para uma assistência com qualidade.

É oportuno ainda, valorizarmos a sugestão dada durante a pesquisa de se abrir espaço para a inclusão das lesões nos quadros de comunicação sobre o paciente, gerando uma estratégia para a prevenção e acompanhamento das lesões de pele, incluindo mais uma meta da segurança do paciente "reduzir o risco de lesões de pele" ao quadro.

Ressaltamos que cabe a cada instituição reconhecer suas fragilidades e procurar estratégias que visam a diminuição do risco ao evento adverso, procurando contemplar as ações que priorizam a segurança do paciente. O líder da equipe é o responsável por promover o desenvolvimento dos membros e facilitar os processos de comunicação, conforme rotinas institucionais e deixando claro a importância de cada membro da equipe, além de identificar a necessidade de treinamentos comportamentais para seus liderados. Não basta dizer que é preciso melhorar a comunicação entre as pessoas, as equipes envolvidas com os serviços de Saúde precisam compreender que processos de comunicação padronizados diminuem os riscos de danos aos pacientes.

\section{REFERÊNCIAS}

BEZERRA, A.L.Q. A Segurança do Paciente e a Enfermagem. Revista Nursing, 2018; 21 (239): 2047 2091. Disponível em: < http://www.revistanursing.com.br/revistas/239Abril2018/editorial.pdf> . Acesso em: 15/05/2021.

BRASIL, Ministério da Saúde. Documento de Referência para o Programa Nacional de Segurança do Paciente / Ministério da Saúde; Fundação Oswaldo Cruz; Agência Nacional de Vigilância Sanitária. Biblioteca Virtual em Saúde do Ministério da Saúde, Brasília, 2014.

BRASIL, Ministério da Educação. Metas Internacionais de Segurança do Paciente. Brasilia, 2021. Disponível em: https://www.gov.br/ebserh/pt-br/hospitaisuniversitarios/regiao-sudeste/hc-ufmg/saude/metas-internacionais-de-seguranca-dopaciente/metas-internacionais-de-seguranca-do-paciente.

BRASIL, Ministério da Saúde. Resolução n 36, de 25 de julho de 2013. Institui ações para a segurança do paciente em serviços de saúde e dá outras providências. Diário Oficial da União 25 jul 2013; Seção 1.

BRASIL, Ministério da Saúde. Resolução $n^{0}$ 529, de $1^{\circ}$ de abril de 2013. Institui o Programa Nacional de Segurança do Paciente (PNSP). Diário Oficial da União 02 abr 2013.

BRASIL, Ministério da Saúde. Resolução no 63, de 25 de novembro de 2011. Dispõe sobre os Requisitos de Boas Práticas de Funcionamento para os Serviços de Saúde. Diário Oficial da União 25 nov 2011; Seção 1.B. 
BRASIL, Ministério do Trabalho. Mercado de Trabalho, Conjuntura e Análise / edição especial COVID 19. Instituto de Pesquisa Econômica Aplicada, Brasilia, 2020.

COFEN, Conselho Federal de Enfermagem. DECRETO N 94.406/87, dispõe sobre o exercício da Enfermagem, e dá outras providências. Portal COFEN. Disponível em: http://www.cofen.gov.br/decreto-n-9440687_4173.html

FIOCRUZ, Fundação Oswaldo Cruz: uma instituição a serviço da vida. Pesquisa inédita traça perfil da enfermagem no Brasil. Agência Fiocruz de Notícias, Brasília 2015. Disponível em: https://portal.fiocruz.br/noticia/pesquisa-inedita-traca-perfil-da-enfermagemno-brasil.

LEMOS, C. S., SILVA, K.C. O Uso da Identificação de Pacientes em uma Unidade Hospitalar. Revista de enfermagem UFPE on line., Recife, v.11, n.1, p.130-9, 2017.

NASCIMENTO, E.R.P; TRENTINI, M. O cuidado de enfermagem na unidade de terapia intensiva (UTI): Teoria Humanística de Paterson e Zderad. Revista Latino Americana de Enfermagem, 12:250-7. 2004. Disponível em: http://dx.doi.org/10.1590/S010411692004000200015 . Acesso em: 23/05/2021.

OLINO, L; GONÇALVES, A.C; STRADA, J.K.R; VIEIRA, L.B; MACHADO, M.L.P; MOLINA, K.L; COGO, A.L.P. Comunicação efetiva para a segurança do paciente: nota de transferência e Modified Early Warning Score. Revista Gaúcha de Enfermagem, v.40 (spe), 2019.

SILVA, A.E.B.C.Segurança do paciente: desafios para a prática e a investigação em enfermagem. Revista Eletrônica Enfermagem, v.12, n.3, 2010. Disponível em: www.revistas.ufg.br/index.php/fen/article/view/11885/7815

WHO, World Health Organization: World Alliance for Patient Safety, Taxonomy: The Conceptual Framework for the International Classification for Patient Safety: final technical report. Genebra; 2009

Publicado em 24/02/2022 